\title{
Spider Search Algorithms for MIMO System and Assessment Using Simatic PCS7
}

\author{
Vijay Anand J. ${ }^{*}$, Manoharan P. S., Jeyadheep Vignesh J., Varatharajan M., Rubina Sherin M.
}

\begin{abstract}
This paper shows two optimization methods that are built on a spider optimization algorithm to enhance the proportional integral and derivative (PID) gain values for multiple-input-multiple-output (MIMO) arrangement which is automated with SIMATIC PCS7 Distributed Control System (SDCS). The leading methodologies are the Spider Search Algorithm (SSA) and Social Spider Optimization (SSO) which is meant primarily for optimizing PID gain values. The SSA is based on foraging strategy of colonial spiders and SSO works on the combined plan of the male and female spiders that removes the episodes of local optimization and exploration elusion. Thus, SSA and SSO are contrived for the ideal fine-tuning of PID conditions in the benchmark MIMO procedure. The system performance is understood by minimizing the integral absolute error (IAE) and the integral square error (ISE) as its objective functions. The time-domain features are examined for the aforesaid methods and thereafter compared with the previous genetic algorithm (GA). The settling time is $60 \mathrm{~s}$ for the proposed method which is lesser than the other techniques. For illustrating the implemented controller's strength, interference is manually presented in the real-time system. Findings indicate that the SSO surpasses output measures and performance indices beyond the presupposed SSA and GA intervals.
\end{abstract}

Keywords: Adaptive control; Optimizing PID gains; Simatic PCS7; Social Spider Optimization; Spider Search Algorithm; Simatic manager

\section{INTRODUCTION}

Most of the processes in the real world are time delays and the delays are mainly caused by delays in acquiring the device's data and interaction delays. The emergence of gaps in time poses further problems for implementation of process control. Therefore, process industries need automation in order to enhance manufacturing quality, productivity, reducing the rate of refusal of products; and adhering fully with safety and environmental regulations.

MIMO process is extensively employed in amalgamation processes in chemical, pharmaceutical food and beverage industries for mixing and blending works. This cumbersome procedure is nonlinear while preserving the levels in tanks at different operating areas. Many variables have a major impact on the system, such as environmental conditions, type of chemical agent, temperature, level and speed of circulating water, etc. Manual and semi-automatic operator stations are being used by the existing process industries; however, the real level of technology is at a lower level. The technicians should therefore enhance their skills in real-time systems.

Most industrial control systems use PID controllers because of their simplicity and flexibility. They can calculate the error which is the contrast between the specified reference point and the variable measured. Another approach in PID tuning is using optimization methods. Different PID optimization strategies have been proposed for the chaotic time delay systems. Some of the objective functions include, Integral Square Error (ISE), Integral Absolute Error (IAE), Integral time square error (ITSE), Integral time Absolute Error (ITSE) etc. Among the bioinspired algorithms, this paper formulates two optimization methods namely SSO and SSA to solve the optimization problem. The aim of this work is to provide a viable alternative for optimizing PID gain values using the SSO and SSA methods for MIMO process plants.

The proposed algorithms should minimize the IAE and ISE which provides sustenance for reliable operation, and resilience for industrial applications. In this research work, the experimental configuration is interfaced with the SDCS by using distributed input-output module (ET200M) and
Industrial Ethernet bus for engineering/operator stations. The S7 Simatic Manger software is used to establish control logics and interaction with all appliances through TCP/IP protocol with the tank system.

\section{LITERATURE SURVEY}

Some methods have been used for tuning the PID controller including Ziegler Nichols (ZN) method, Cohencoon method etc. These methods produce quadrature decay ratio response at the output. The response does not augur well for processes in the industrial environment. An easy and effective control strategy for stable and chaotic first order systems with time delay was proposed, and to achieve better performance PID controller with second order lead / lag filter was used in [1]. The tuning of PID controllers for process control industries has been detailed in [2-7].

PID tuning problem has been converted into optimization problem in which the controller parameters have been chosen in such a way that ensures the optimization of an objective function. Several types of evolutionary algorithms (EAs) which come under evolutionary computing have been used widely to solve real time optimization problems. EAs demonstrated and performed better than the conventional optimization techniques, particularly in solving nonlinear optimization problems [810]. A few bio-inspired optimization techniques were incorporated as below:

- Genetic Algorithm (GA),

- Particle Swarm Optimization (PSO),

- Ant Colony Optimization (ACO),

- Spider Search Algorithm (SSA),

- Social Spider Optimization Algorithm (SSO).

The GA is well known for its role in reducing computational efforts and providing rapid solutions optimally for the non-linear systems. Hence GA was taken here to analyze and collate the results with SSA and SSO. Various algorithms have been discussed wherein they inspired to develop and implement practical applications [11]. Ant colony optimization has been traversed into a problem of finding the superlative solutions in a bounteous path using their search patterns [12]. Researchers developed 
optimization algorithms by imitating artificial bee [13, 14] and swarm optimization technique [15].

The paper discussed spider search algorithm [16, 17] based on its prey catching method and developed an algorithm with spider logic which was implemented on various benchmark functions. The algorithm is based on the spider's social co-operative behavior in the communal web. The model and design of interacting and non-interacting tank systems for a real time application using various controllers have been discussed $[18,19]$.

The use of PCS 7 DCS in the industry makes the process efficient and reduces manpower to a greater constraint. The reliability of the multipurpose manipulator has been regulated by the Siemens PCS7 Distributed Control System (DCS). A chemical reactor was controlled remotely and tested in the laboratory of undergraduate department of chemical engineering. The water pump model for manufacturing plants with field bus communication protocols in a laboratory was automated using Simatic Manager and IWAN network [20-23]. The hardware and software configurations made use of user manuals [24, 25].

From the research work reviewed, it became evident that PID controllers were widely used in many industrial control systems because of the simplicity of structure and the ease of implementation. Since then, only a few papers addressed the automation part using distributed control system, especially the SDCS for practical applications. Hence, this paper illustrates the SDCS automation for MIMO systems by optimizing the PID gain values using SSO and SSA optimization algorithms.

\section{$3 \quad$ PROPOSED METHODOLOGIES}

The aforesaid methods are used to minimize the IAE and ISE of the MIMO model in the PID controller for attaining the optimum gain values. The PID controller gain values are calibrated to produce the desired outcomes by using SSO and SSA. Fig. 1 shows the system block diagram designed in accordance with ambient conditions $\left(35^{\circ} \mathrm{C}\right.$ and atmospheric pressure) and the desired process control. The detailed modelling overview, interfacing SDCS and the SSO algorithm design for the MIMO model is presented in the upcoming chapters.

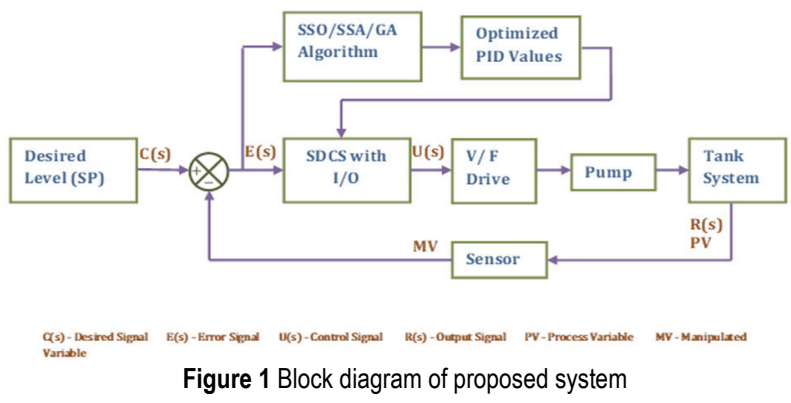

\subsection{Spider Search Algorithm}

The algorithm is based on the spider's behavior in search of its prey. The spider acts as an agent in SSA. The number of the spider counts placed on the spider web is predefined in the algorithm. In this work, the minimum value for IAE is attained by tuning the gain values $(K)$ of PID parameters $\left(K_{p}, K_{i}, K_{d}\right)$ which are considered as spiders. Vibration plays an important role in SSA and it is one of the most important features that distinguish SSA from other mimetic algorithms. Vibration can be defined with two properties; namely, the source position and the source vibration strength.

The vibration strength in the source of the spider location is associated with the fitness of its location $f\left(L_{s}\right)$. The vibration strength value is presented below in Eq. (1).

$$
I\left(L_{s}, L_{s}, t\right)=\log \left(\frac{1}{f\left(L_{s}-c\right)}+1\right)
$$

The distance between two spiders is defined as $D\left(L_{a}\right.$, $\left.L_{b}\right)$ and by using the Manhattan distance which is one norm to determine the distance as mentioned in Eq. (2).

$D\left(L_{a}, L_{b}\right)=\left\|L_{a}-L_{b}\right\|$

By taking the standard deviation of the entire spider's position, the attenuation of the vibration over distance is determined as in Eq. (3).

$$
I\left(L_{a}, L_{b}, t\right)=I\left(L_{a}, L_{a}, t\right) \cdot \exp \left(-\frac{D\left(L_{a}, L_{b}\right)}{\bar{\sigma} \cdot r_{a}}\right)
$$

In the searching technique of the SSA, there are three stages namely initialization, iteration and final. In every SSA run, it starts by initializing the stage and then performing the search in an iterative manner. Finally, the iteration will stop when the halting criteria are satisfied in the maximum boundary. The algorithm produces the best solution with the best fitness found after the iteration.

The algorithm was run for around 30 iterations for SSA in MATLAB software. From the iterations, the gain values $\left(K_{P}, K_{I}\right.$ and $\left.K_{D}\right)$ were taken according to the least IAE and ISE values to simulate the system for the closed loop response. Before testing it with real plant, the same procedure was done for the transfer function of real time system in MATLAB simulation.

\subsection{Social Spider Optimization Algorithm}

SSO is chosen because it demonstrates its collaborative strategy with the male and female spiders. The algorithm is predicated on the co-operative behavior of the social spiders, using their vibrations on web. The spider has similar walking strategies when comparing with other species. It can change its position as quickly as possible and hit the target faster. In the function of determining the optimal value for the PID parameters, this approach is used to reach the target. Several times the SSO method has been employed to get the converged PID parameters and the primary focus of applying the SSO method to minimize the IAE and ISE values.

\subsubsection{The Concept and Structure of the proposed method}

Extensive research involved the design of robots based on a spider walking pattern. Scientists deduced that some of the spiders remained social in 35.000 spider families. The algorithm was developed on the basis of the character 
of a social spider. SSO Algorithm is used in finding the optimal solutions in non-linear systems. The number of male and female spider counts on the spider's web is predefined in the algorithm. The spider clustering and its search space are limited to its web network and the spider's individual spot rivalry for a possible solution that is analogous to the optimization problem. The Spider campaign is controlled by three different search criteria leading to optimal global solutions. The total population contains 65 to $90 \%$ of female spiders and the rest are male spiders.

The initial step towards the algorithm is initializing the population accurately. This is shown below.

$f_{s}=(0.9-$ rand $\cdot 0.25) \cdot K$

$M_{s}=K-f_{s}$

where $N$ defines the total population of the spiders, $f_{s}$ denotes the female spiders and rand is a random number between 0 and $1 . M_{s}$ denotes the male spiders and it is derived from the difference between $N$ and $f_{s}$.

The fitness function is based on the weight that has been assigned to each spider.

$W_{i}=\frac{J\left(s_{i}\right)-\text { worst }_{s}}{\text { best }_{s}-\text { worst }_{s}}$

where $J\left(s_{i}\right)$ represents the fitness value obtained by the evaluation of the spider position $s_{i}$ with regard to the objective function.

The most effective form of spider communication is vibration. Each spider transmits and receives the vibrations by relying on the weight of the spider. The stronger vibration is perceived by the spider's weight whereby one spider gets either attracted or repelled.

$V i b_{i j}=w(j) \cdot e^{-d^{2} i, j}$

where $d_{i, j}$ is the Euclidian distance between the spiders $i$ and $j$.

The search technique of the spiders is cleaved into three parts. Firstly, the Spider $s_{i}$ perceives the information that is dispatched by the Spider $s_{c}$ that is nearer and heavier:

$V i b_{i c}=w(b) \cdot e^{-d^{2} i, c f}$

Secondly, the Spider $s_{i}$, perceives the information that is dispatched by the Spider $s_{b}$ that holds the best weight of the entire population $S$ :

$V i b_{i b}=w(b) \cdot e^{-d^{2} i, b}$

Thirdly, the Spider $s_{b}$ perceives the information that is dispatched by the Spider $s_{f}$ which is a female spider that is nearer:
$V i b_{i f}=w(b) \cdot e^{-d^{2} i, f}$

The female spiders are the mating agents. Their attraction is based on the weight and the distance of the other spider in the communal web.

$F_{i}^{K+1}=F_{i}^{k}+\alpha \cdot v_{c i}\left(s_{c}-F_{i}^{k}\right)+\beta \cdot v_{b i}\left(s_{b}-F_{i}^{k}\right)+\delta \cdot\left(\operatorname{rand}-\frac{1}{2}\right)$

where $v i b_{c i}$ is the vibrations perceived by the spider $s_{c}$ which holds the higher weight and that is nearer to the source spider.

$v i b_{b i}$ indicates the movement of the female spider towards the best spider in the entire population.

rand indicates the random movement. This movement is possible when $r_{m}<p f$.

When the above condition is not satisfied the movement of the female spider tends to be:

$F_{i}^{K+1}=F_{i}^{k}-\alpha \cdot v_{c i}\left(s_{c}-F_{i}^{k}\right)-\beta \cdot v_{b i}\left(s_{b}-F_{i}^{k}\right)+\delta \cdot\left(\operatorname{rand}-\frac{1}{2}\right)$

There are two types in male spider community, namely Dominant and Non-dominant spiders. The dominant spiders are the ones with the essential fitness attribute that dominates the female spider, while the non-dominant males are not up to the mark, they tend to reach the male spider's mid-position.

When the male is dominant:

$M_{i}^{K+1}=M_{i}^{k}+\alpha \cdot v i b_{f i}\left(s_{f}-M_{i}^{k}\right)+\delta \cdot\left(\operatorname{rand}-\frac{1}{2}\right)$

For the non-dominant male spiders their movement is guided by:

$M_{i}^{k+1}=M_{i}^{k}+\alpha \cdot\left(\frac{\sum_{h=1}^{N_{m}} M_{h}^{k} *_{N_{f+h}}}{\sum_{h=1}^{N_{m}} w_{N_{f+h}}}-M_{i}^{k}\right)$

Thus, the spider's movement is directed to the grounds of this search. When the higher-weight male spider is attracted to the female spider, a new spider will be created. The new spider is formed by means of the roulette formation. Next, the new spider that is formed is compared to the worst spider. The search continues until an optimized solution is obtained. The formation of spiders and how it reaches the global optimum was shown in the Fig. 2.

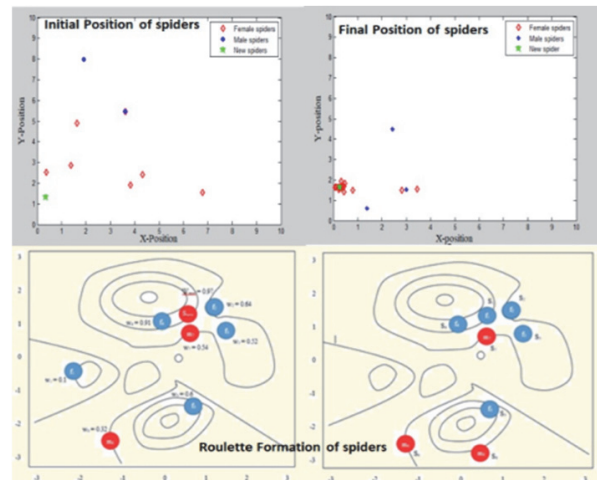

Figure 2 Formation and position of spiders 
The formation of a new spider is determined by the spiders holding the heavier weight and the probability of it is defined by:

$$
P s_{i}=\frac{w_{i}}{\sum_{j \in T} w_{j}}
$$

Table 1 Roulette Formation and new spider calculation
\begin{tabular}{|c|c|c|c|c|}
\hline \multicolumn{2}{|c|}{ Spider } & Position & $w_{i}$ & $P_{s i}$ \\
\hline$S_{1}$ & $f_{1}$ & $(-1.9,0.3)$ & 0.1 & - \\
\hline$S_{2}$ & $f_{2}$ & $(1.4,1.1)$ & 0.64 & 0.24 \\
\hline$S_{3}$ & $f_{3}$ & $(1.5,0.2)$ & 0.52 & 0.19 \\
\hline$S_{4}$ & $f_{4}$ & $(0.4,1.0)$ & 0.92 & 0.34 \\
\hline$S_{5}$ & $f_{5}$ & $(1.0,-1.5)$ & 0.61 & - \\
\hline$S_{6}$ & $m_{1}$ & $(-1.3,-1.9)$ & 0.32 & - \\
\hline$S_{7}$ & $m_{2}$ & $(0.9,0.7)$ & 0.54 & 0.20 \\
\hline$S_{8}$ & $m_{3}$ & $(0.8,-2.6)$ & 0.44 & - \\
\hline$S_{\text {new }}$ & & $(0.9,1.1)$ & 0.97 & - \\
\hline
\end{tabular}

$\Sigma P S_{i}=0.24+0.19+0.34+0.20=0.97$

Weight of the new spider is 0.97 and $f_{1}$ is discarded since the newly formed spider has higher weight than the worst spider.

\section{SDCS CONFIGURATION}

The system comprises two main sections, namely hardware and software. By considering environmental conditions of industries, system design, control and connectivity mechanisms are developed for decentralized processes in the field level. In this work, 2 analog inputs (AI) and 2 analog outputs (AO) are used. The number of inputs and outputs may be increased, and this will be operated with additional modules. Fig. 3 shows the general view of SDCS in the DCS Integrated Engineering laboratory of Thiagarajar College of Engineering (TCE), Madurai.

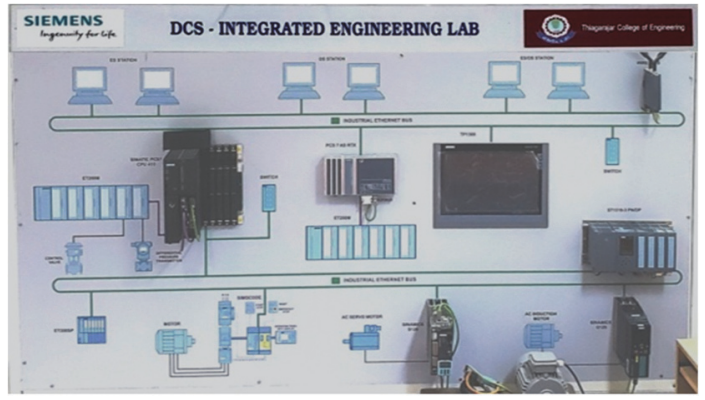

(a)

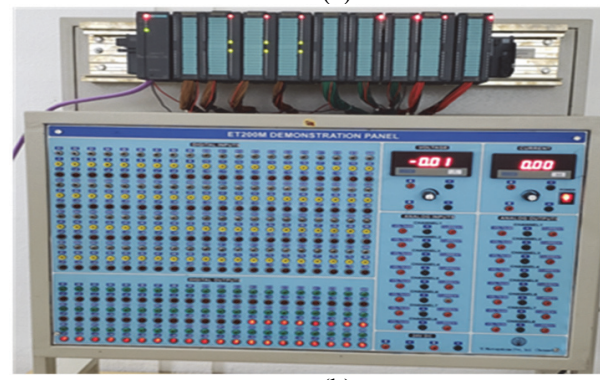

(b)

Figure 3 General View of SDCS: Access Node (a) and ET200M I/O Module (b)

\subsection{Hardware Configuration}

SDCS uses a process administrator to build process documents and preserve them. There are two primary elements in the hardware configuration. The first component is the computer and multi-point connection that allows the computer to communicate with the SDCS. Level transmitter (LT), PROFINET I/O (PNIO) and PROFIBUS DP supports the correlation of field devices to SDCS. The second component is the PNIO system which combines engineering stations and operator stations. For connecting SDCS processing inputs, a separate modular with extremely flexible DP slave ET $200 \mathrm{M} \mathrm{I} / \mathrm{O}$ unit is used. This module was chosen to support PROFIBUS DP and PNIO buses. For the PROFINET IO bus, the ET200MIM153-2 standard with 4 digital inputs, 3 digital outputs, 1 analog Input, 1 analog output with a $24 \mathrm{~V}$ power module are used in this work. Fig. 4, Fig. 5 and Fig. 6 show the hardware configuration of SDCS in S7 Simatic manager platform.

\subsection{Software Configuration}

Two main software configurations are used in this work.

The SIMATIC MANAGER software is used for designing the function library such as continuous flow chart (CFC) to develop control logic, algorithms and WinCC (SIMATIC Windows Control Center). This is a graphical user interface (GUI) to establish interconnections within a project i.e. simulation of the user interface and system. The WinCC GUI enables integrated deployment with Simatic Manager up to PC-based visualizing workstations for all SIMATIC operator panels.

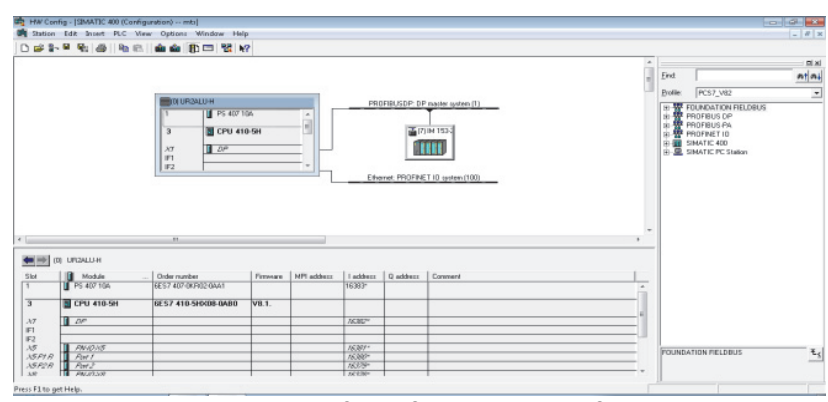

Figure 4 Ethernet and PROFIBUS management in Simatic Manager

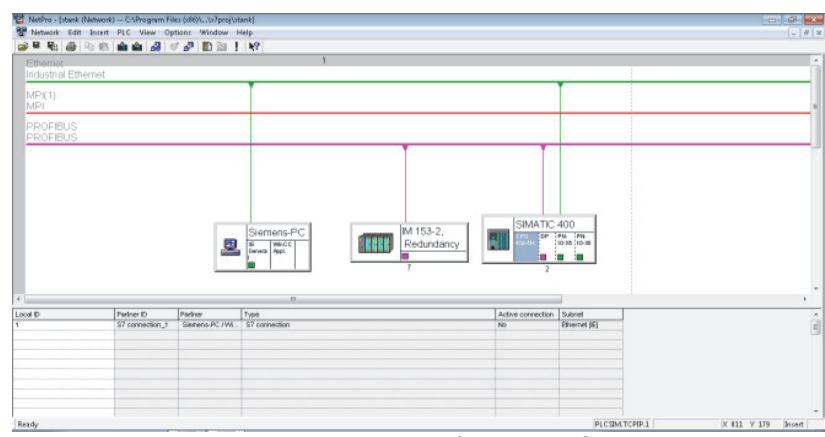

Figure 5 Hardware and network configuration in Simatic Manager 


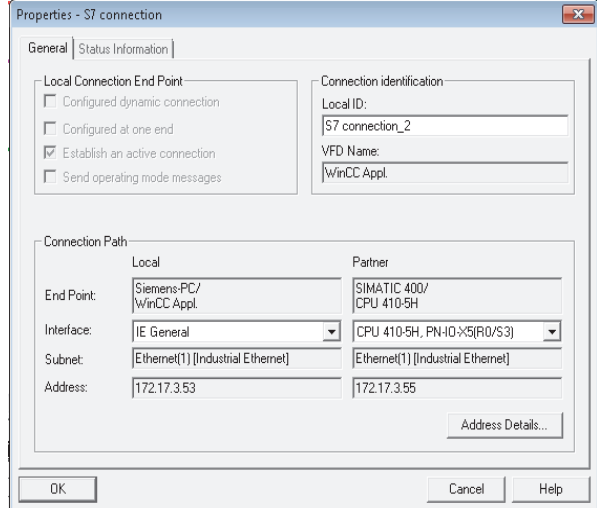

Figure 6 Operator Station interfaced with SDCS

\section{MATHEMATICAL MODELLING of MIMO PROCESS}

Fig. 7 shows the schematic diagram of the two-tank interacting system configured as a MIMO system. The Tanks T1, T2 are cylindrical and indistinguishable. These two tanks are intertwined by a physically controlled valve, while VFD 1and VFD 2 are the two voltage frequency drives for the tanks $\mathrm{T} 1$ and $\mathrm{T} 2$ respectively.

The $\mathrm{T} 1$ and $\mathrm{T} 2$ tank levels are referred to as $\mathrm{H} 1$ and $\mathrm{H} 2$ respectively. The liquid height is relayed in the form of 4 $20 \mathrm{~mA}$. The LT1 and LT2 pressure transmitters are used to gauge the level based on the pressure accumulated by the liquid in the tank.

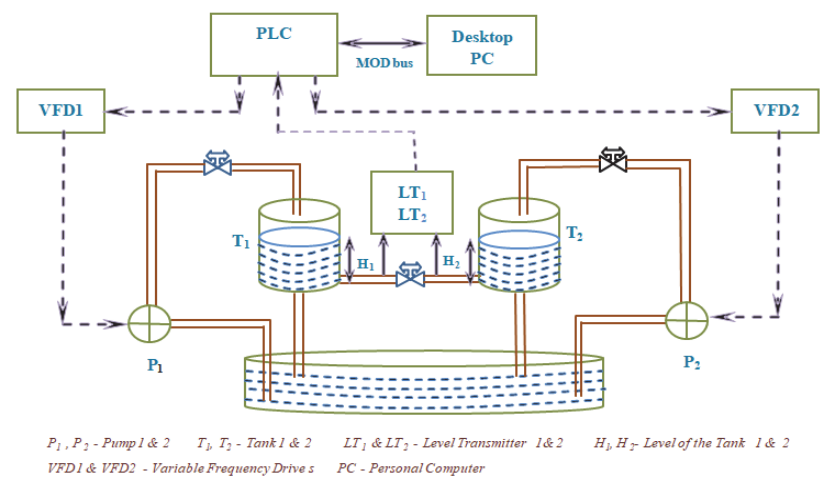

Figure 7 Schematic diagram of MIMO system

Since the controlled and manipulated variables for the interacting mode are two, four transfer functions were required to fully represent the process dynamics. The tank levels were first maintained at a level of $50 \%$ by giving 25 $\mathrm{Hz}$ to the voltage frequency drives as input for the pumps $P_{1}$ and $P_{2}$ respectively. The input to the VFD1 was then adjusted from $25 \mathrm{~Hz}$ to $30 \mathrm{~Hz}$ by maintaining VFD2 as constant and tracking the tank's levels. The same process is performed by adjusting the VFD2 input from $25 \mathrm{~Hz}$ to 30 $\mathrm{Hz}$ while maintaining the VFD1 input as a constant. The general equation for the transfer function of a MIMO model is given in Eq. (17).

$$
G(s)=\left[\begin{array}{ll}
g_{11} & g_{12} \\
g_{21} & g_{22}
\end{array}\right]
$$

The open loop plot acquired by the experiment has been shown in Fig. 8. Using the system identification tool box, the transfer function of the MIMO process is described below.

$$
G(s)=\left[\begin{array}{cc}
\frac{0.578 \mathrm{e}^{-39.5 s}}{14.14 s+1} & \frac{0.0693 \mathrm{e}^{-40 s}}{11.29 s+1} \\
\frac{0.0692 \mathrm{e}^{-39.7 s}}{11.21 s+1} & \frac{0.0707 \mathrm{e}^{-40 s}}{11.86 s+1}
\end{array}\right]
$$
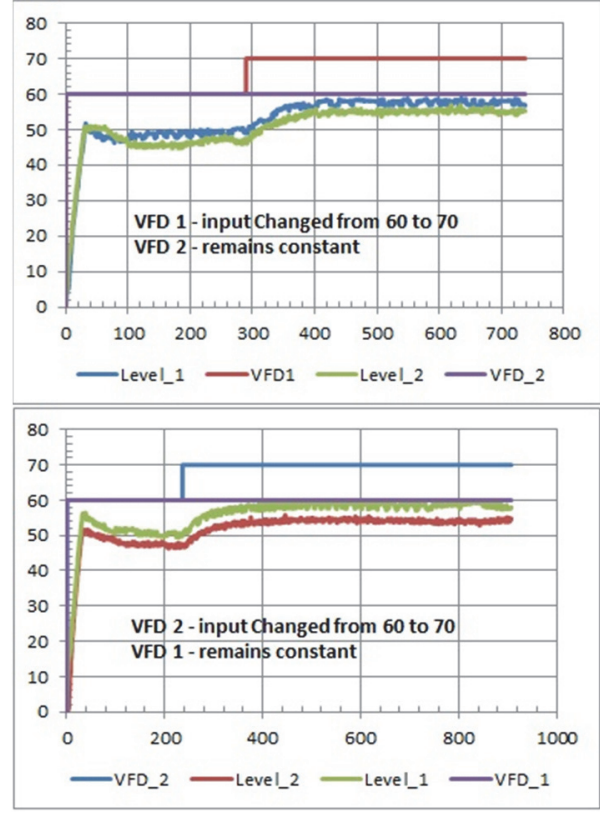

Figure 8 Open-Loop response of the system with interacting mode

\section{SIMULATION RESULTS AND DISCUSSION 6.1 Empirical PID Tuning Algorithms}

The modelled system was first simulated and experimented with the empirical PID tuning techniques such as Ziegler-Nichols (ZN), Cohen-Conn (CC), ChienHrones-Reswick (CHR), and Wang-Juang-Chan (WJC). The calculated PID parameters for these tuning techniques are tabulated in Tab. 2 and their responses are also plotted in Fig. 9. From the response it is shown that the empirical tuning methods produce steady state error and overshoots.

Table 2 PID parameters, IAE and ISE values for SSO, SSA and GA

\begin{tabular}{|c|c|c|c|}
\hline & $K_{P}$ & $\tau_{i}$ & $\tau_{d}$ \\
\hline ZN (Open Loop) & 7.4268 & 0.3800 & 1.5200 \\
\hline ZN (Closed Loop) & 7.5600 & 0.3500 & 1.400 \\
\hline Cohen-Coon & 7.7720 & 0.2619 & 1.7325 \\
\hline CHR - 0\% overshoot & 3.7134 & 0.3800 & 1.9600 \\
\hline CHR - 20\% overshoot & 5.8795 & 0.3572 & 2.7440 \\
\hline WJC & 4.3334 & 0.3183 & 2.3400 \\
\hline
\end{tabular}

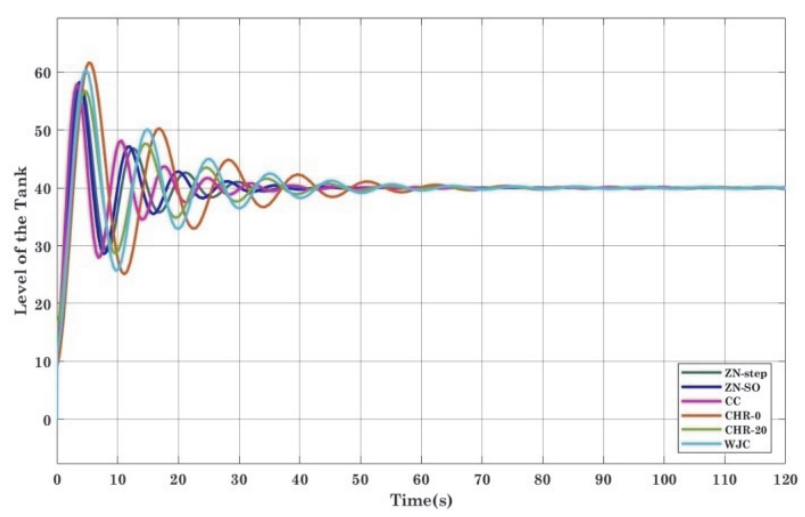

Figure 9 Closed loop responses of various empirical tuning approaches 


\subsection{MIMO Process Simulation Results}

The SSO, SSA and GA approaches were run for the transfer function of the actual plant with the cross coupled PID technique.

Fig. 10 represents the simulation diagram of the plant with the controller. In this process, cross coupled PID control was used as the centralized controller. Fig. 10 represents the output of the MIMO process for their respective optimized gain values which is taken from the simulation. During simulation the disturbance was added and it was reflected in the graph.

The time domain specifications for all the three algorithms are illustrated in Tab. 3. The performance indices (mean, standard deviation and variance) were calculated for the IAE and ISE values which are mentioned in Tab. 4. Interestingly, these indices inferred that the SSO algorithm worked in an efficient way when compared to SSA and GA method.

Table 3 Time domain specifications for MIMO system

\begin{tabular}{|c|c|c|c|}
\hline Controllers & $\begin{array}{c}\text { Rise Time } T_{R} \\
/ \mathrm{s}\end{array}$ & $\begin{array}{c}\text { Peak Overshoot } M_{p} / \\
\%\end{array}$ & $\begin{array}{c}\text { Settling time } T_{S} / \\
\mathrm{s}\end{array}$ \\
\hline GA & 11 & 0.15 & 160 \\
\hline SSA & 12 & 0.001 & 100 \\
\hline SSO & 12 & 0.001 & 60 \\
\hline
\end{tabular}

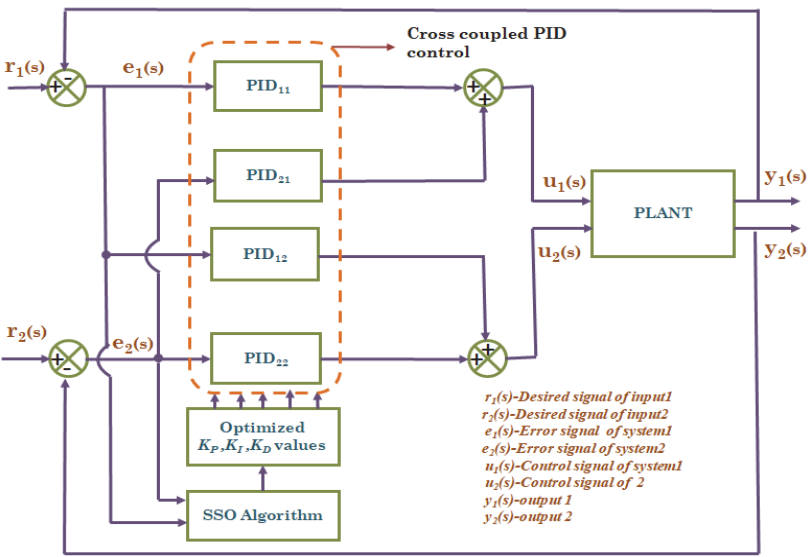

Figure 10 Simulation diagram of interacting tank system
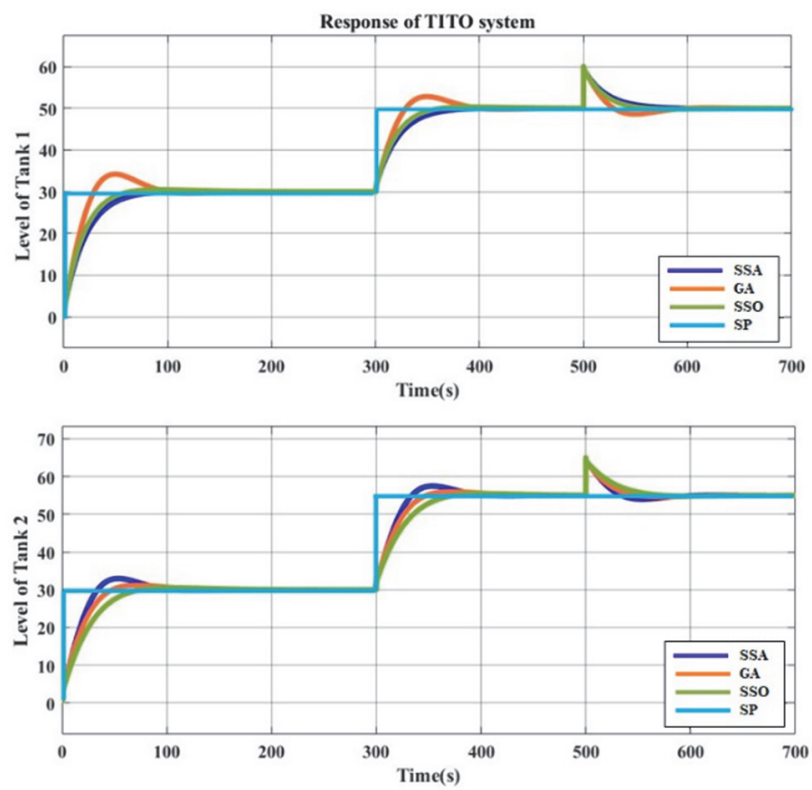

Figure 11 Simulation diagram of interacting tank system
Table 4 Comparison of IAE Values for SSO, SSA and GA

\begin{tabular}{|c|c|c|c|c|}
\hline \multicolumn{2}{|c|}{ Performance Indices } & $\begin{array}{c}\text { Genetic } \\
\text { Algorithm }\end{array}$ & SSA & SSO \\
\hline \multirow{3}{*}{ IAE } & $\begin{array}{c}\text { Standard } \\
\text { Deviation }\end{array}$ & 0.15473 & 0.14453 & 0.00232 \\
\cline { 2 - 5 } & Mean & 0.15743 & 0.12783 & 0.01263 \\
\cline { 2 - 5 } & Variance & 0.00684 & 0.00349 & 0.00245 \\
\hline \multirow{3}{*}{ ISE } & $\begin{array}{c}\text { Standard } \\
\text { deviation }\end{array}$ & 0.29743 & 0.29453 & 0.29133 \\
\cline { 2 - 5 } & Mean & 0.29243 & 0.28782 & 0.28693 \\
\cline { 2 - 5 } & Variance & 0.08648 & 0.08439 & 0.08437 \\
\hline
\end{tabular}

\section{EXPERIMENTAL SETUP OF TANK SYSTEM}

The experimental setup of the multi-tank system and its components is depicted in Fig. 12. In this setup, the bottom two tanks are configured for MIMO process.

- The height of the cylindrical tank (T1 and T2) is $30 \mathrm{~cm}$ each.

- The pumps $\left(\mathrm{P}_{1}, \mathrm{P}_{2}\right)$ are operated by the voltage frequency drives $\left(\mathrm{VFD}_{1}\right.$ and $\left.\mathrm{VFD}_{2}\right)$.

- The levels of the tanks are measured by the pressure transmitters $\left(\mathrm{LT}_{1}, \mathrm{LT}_{2}\right)$.

- The protocol TCP/IP is used for communication through Profibus and Industrial Ethernet.

- S7 Simatic Manager software is used to interface the tank system.

- The entire tank system is installed in Control System Laboratory in TCE campus next to the Siemens Automation Laboratory.

- The SDCS was interfaced to the hardware setup by Profibus through the distributed I/O module. The Profibus is a small independent unit used in process control systems. It ensures that system data communication is highly reliable, non-blocking and high speed.

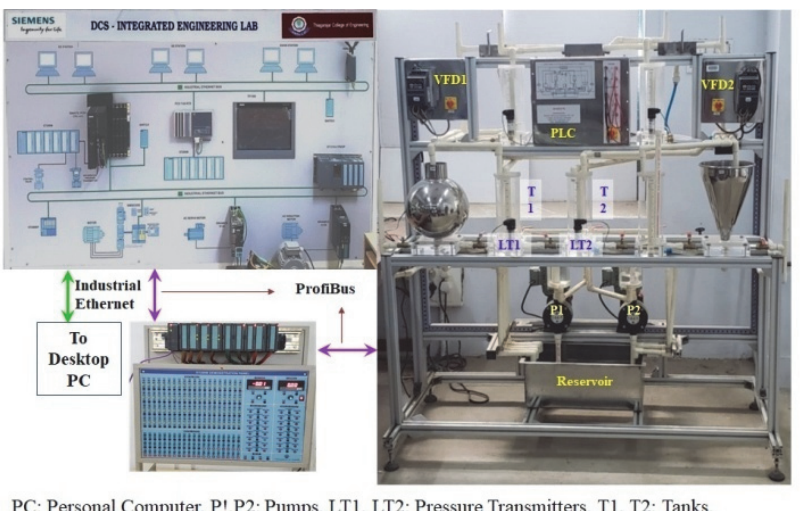

PC: Personal Computer, P!,P2: Pumps, LT1, LT2: Pressure Transmitters, T1, T2: Tanks, VFD1, VFD2: Variable Frequency Drives, PLC: Programmable Logic Controller

Figure 12 Experimental Setup of the time delay process system

\subsection{Experimental Results}

The control logic for the process is developed in CFC as shown in Fig. 13. The system uses the analog input module $\mathrm{CH}$ AI, analog output module $\mathrm{CH} \_\mathrm{AO}$ and CTRL PID blocks in the advanced process library function. In this section, two experiments were conducted. First test measures the steady-state effect without disruption.

The other test is to find the anti-disturbance capability of the method, where the disturbance is triggered by adding 
water externally to the tank with measurements 250,350 and $500 \mathrm{ml}$. The gain values of PID have been given respectively to gain form (Fig. 14). CFC is downloaded into SDCS and the process is run continuously. The WinCC GUI's graph is shown in Fig. 15.

The experimental results for MIMO systems by using $\mathrm{ZN}$ and $\mathrm{CC}$ tuning methods are shown in Fig. 16. Fig. 17 shows trend view of the SISO system's closed loop response after output perturbation for the minimum IAE and ISE values using SSO, SSA and GA optimization techniques. The trend value is recorded and the charts are plotted. From the trend, it is shown that the process can transit smoothly to a steady-state by tracking the level curve with the continuous operation. Fig. 18 shows the output plot of the MIMO system's closed loop response for SSO, SSA and GA algorithms. The system returns to the normal state easily and therefore, the system has a relatively strong anti-disturbance quality.

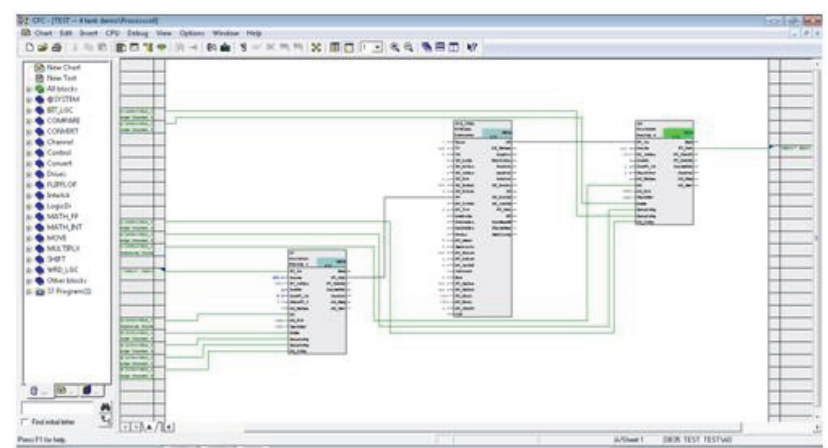

Figure $13 \mathrm{CFC}$ for the pump control

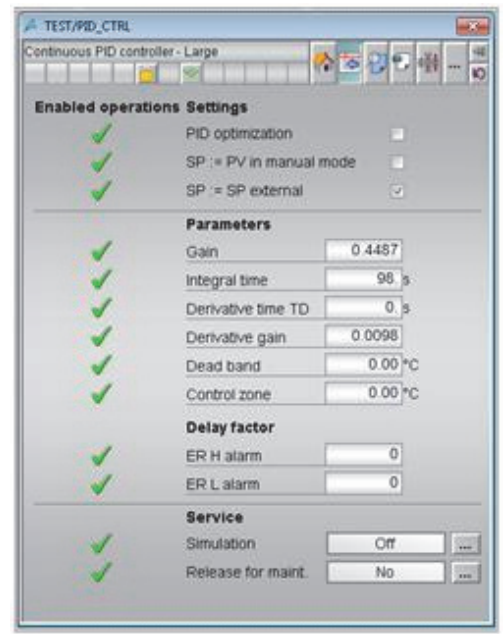

Figure 14 PID Parameterization

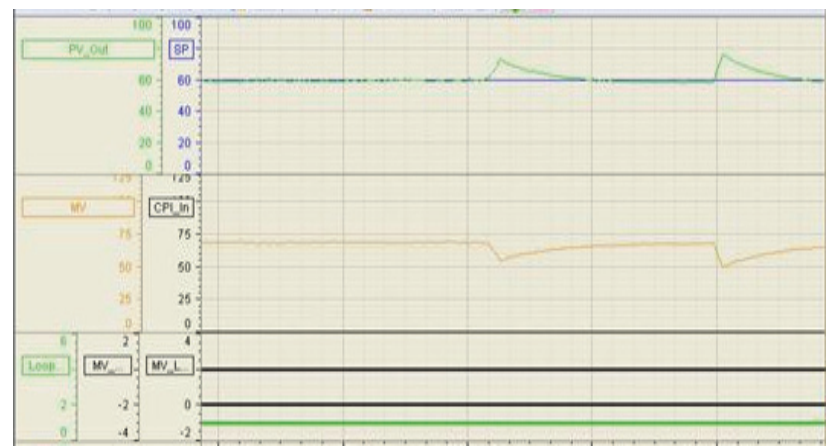

Figure 15 WinCC Faceplate graph view

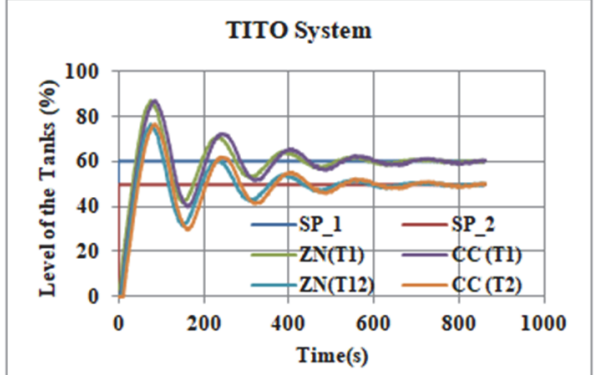

Figure 16 Closed loop response for empirical tuning methods

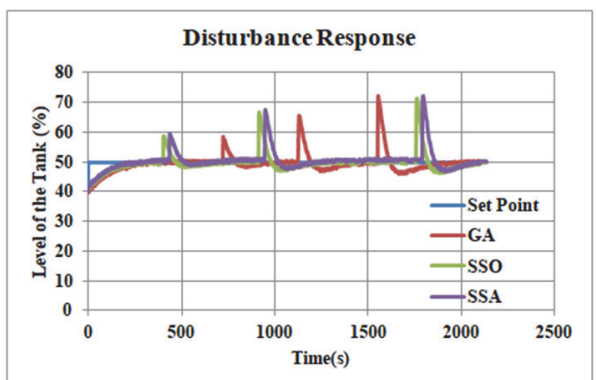

Figure 17 Closed loop response with output perturbation
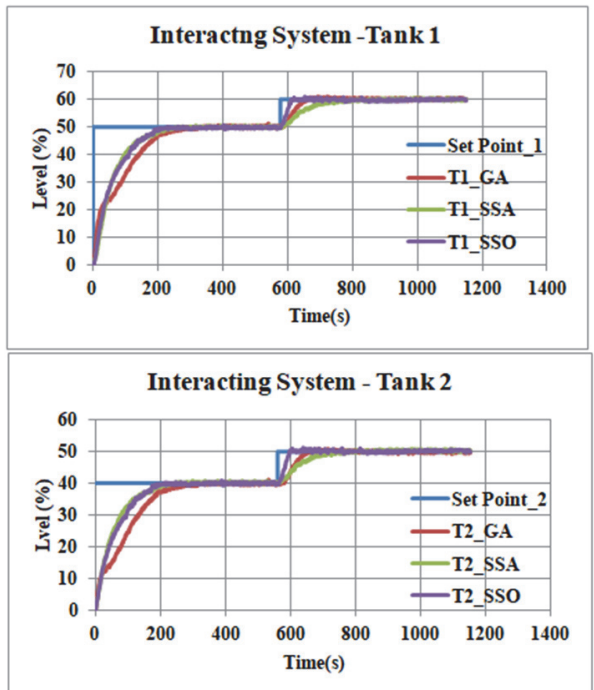

Figure 18 Closed loop response for MIMO process

\section{CONCLUSION}

This paper implemented spider optimization algorithms such as SSA and SSO for optimizing the PID controller gain values in the MIMO process. The main feature of this work was the application of SDCS for a real time tank system. The adopted optimization methods were analysed for the first order with dead time (FODT) transfer function model in the real time system. The plant was also assessed with empirical tuning methods and with GA algorithm. Statistical performance measures such as mean, variance and standard deviation along with time domain points such as rise time, settling time and maximum peak overshoot were evaluated. By analyzing the graphical results and analysis, some of the main features of this experiment are marked as the following:

- The simulation and hardware results proved that IAE and ISE values of SSO were less than SSA and GA. 
- It was marked that the SSO method surpassed SSA and GA methods in performance while bouncing SSO scheme optimum conditions.

- Furthermore, the results displayed a satisfactory tracking effect together with control strategy strength.

- The dispersed monitoring structure that was created using SDCS possessed a prototype for further research in the years to come.

\section{Acknowledgements}

The authors express their gratefulness to Thiagarajar College of Engineering, India for their extensive support. The authors acknowledge IEI R\&D Grant-in-Aid Scheme, The Institute of Engineers (INDIA) (Ref: R.6/2/DR/201920/DR2020015, dated $7^{\text {th }}$ February 2020) for their valuable financial support.

\section{REFERENCES}

[1] Medarametla, P. K. \& Muthukumarasamy, M. (2018). A Novel PID Controller with Second Order Lead/Lag Filter for Stable and Unstable First Order Process with Time Delay. Chemical Product and Process Modeling, 1(13), 1-16. https://doi.org/10.1515/cppm-2017-0010

[2] Bazanella, A. S., Pereira, L. F. A., \& Parraga, A. (2017). A new method for PID tuning including plants without ultimate frequency. IEEE Transactions on Control Systems Technology, 2(25), 637-644. https://doi.org/10.1109/TCST.2016.2557723

[3] Li, K. (2013). PID Tuning for Optimal Closed-Loop Performance with Specified Gain and Phase Margins. IEEE Transactions on Control Systems Technology, 3(21), 10241030. https://doi.org/10.1109/TCST.2012.2198479

[4] Lennartson, B. \& Kristiansson, B. (2009). Evaluation and tuning of robust PID controllers. IET Control Theory \& Applications, 3(3), 294-302. https://doi.org/10.1049/iet-cta:20060450

[5] Antonio, V. (2006). Practical PID Control. Springer.

[6] Shen, J. C. (2002). New tuning method for PID controller. ISA Transaction, 4(41), 473-484. https://doi.org/10.1016/S0019-0578(07)60103-7

[7] Astrom, K. J. \& Hagglund, T, (1995). PID Controllers: Theory Design and Tuning. $2^{\text {nd }}$ Edition, ISA Press Research Triangle Park.

[8] Yang, X. S. (2008). Nature-inspired metaheuristic algorithms, in Fire fly Algorithm. Luniver Press.

[9] El-Ghazali, T. (2009). Metaheuristics from Design to Implementation. John Wiley \& Sons.

[10] Parpinelli, R. S. \& Lopes, H. S. (2011). New inspirations in swarm intelligence: a survey. International Journal of BioInspired Computation, 1(3), 1-16. https://doi.org/10.1504/IJBIC.2011.038700

[11] Cuevas, E., Zaldivar, D., \& Perez-Cisneros, M, (2018). Introduction, in Advances in Metaheuristics Algorithms: Methods and Applications. Studies in Computational Intelligence, $775,1-8$. https://doi.org/10.1007/978-3-319-89309-9_1

[12] Krzysztof, S. \& Marco, D. (2008). Ant colony optimization for continuous domains. European Journal of Operational Research, 3(185), 1155-1173. https://doi.org/10.1016/j.ejor.2006.06.046

[13] Dervis, K. \& Bahriye, B. (2007). A powerful and efficient algorithm for numerical function optimization: artificial bee colony (ABC) algorithm. Journal of Global Optimization, 3(39), 459-471. https://doi.org/10.1007/s10898-007-9149-x
[14] Akay, B. (2013). A study on particle swarm optimization and artificial bee colony algorithms for multilevel thresholding. Applied Soft Computing, 13(6), 3066-3091. https://doi.org/10.1016/j.asoc.2012.03.072

[15] Cuevas, E., Cienfuegos, M., Zaldivar, D., \& Perez-Cisneros, M. (2013). A swarm optimization algorithm inspired in the behavior of the social-spider. Expert Systems with Applications, 16(40), 6374-6384. https://doi.org/10.1016/j.eswa.2013.05.041

[16] James, J. Q. Y. \& Victor, O. K. L. (2015). A social spider algorithm for global optimization. Applied soft computing, 30, 614-627. https://doi.org/10.1016/j.asoc.2015.02.014

[17] Uetz, G. (1992). Foraging strategies of spiders. Trends in Ecology \& Evolution, 7(5), 155-159. https://doi.org/10.1016/0169-5347(92)90209-T

[18] Medewar, P. G., Sonawane, R. R. \& Munje, R. K. (2017). Two Tank Non-Interacting Liquid Level Control Comparison using Fuzzy and PSO Controller. IOSR Journal of Electrical and Electronics Engineering, 24-31.

[19] Kalyanjee, B., Labanya, B., Himadri, D., \& Santu, B. (2015). Study of interacting and non-interacting with disturbance and PID controller design. International Journal of Advanced Computing and Electronics Technology, 3(2), 2394-3416.

[20] Pooventhan, K., Sowndarya, K., Mythily, T., \& Dharhini, V. (2018). Enhancement of Multi-loop Level Control with Optimized Ratio and Split Range Controller using DCS PCS7. International Journal of Scientific \& Engineering Research, 11(9), 1719-1723.

[21] Satheeswaran, G., Hollfeld, K. P., Drews, P., Nicolai, D., Neubauer, O., Schweer, B., \& Grulke, O., (2017). A PCS7based control and safety system for operation of the W7XMulti-Purpose Manipulator facility. Elsevier, Fusion Engineering and Design, 123, 699-702. https://doi.org/10.1016/j.fusengdes.2017.05.125

[22] Maria, L., Botero, A. S., Roger, W., Mukta, B., \& Markus, K. (2016). Cambridge weblabs: A process control system using industrial standard SIMATIC PCS 7. Elsevier, Education for Chemical Engineers, 1-8. https://doi.org/10.1016/J.ECE.2016.04.001

[23] Ramazan, B. \& Yucel, C. (2011). A water pumping control system with a programmable logic controller (PLC) and industrial wireless modules for industrial plants An experimental setup. ISA Transaction, 2(50), 321-328. https://doi.org/10.1016/j.isatra.2010.10.006

[24] Siemens manual, (2017). Configuring Hardware and Communication Connections with STEP 7 Manual.

[25] Siemens manual, (2011). Simatic Process Control System PCS 7 Manual Collections, Advanced Process Library V8.0 Function Manual. 


\section{Contact information:}

Vijay Anand J., Research Scholar (Corresponding author)

Thiagarajar College of Engineering,

Department of Electrical and Electronics Engineering,

Thiruparankundram, Madurai 625015

Tamilnadu, India.

E-mail: jvanandan@gmail.com

Manoharan P. S., Associate Professor

Thiagarajar College of Engineering,

Department of Electrical and Electronics Engineering,

E-mail: psmeee@tce.edu

Jeyadheep Vignesh J., PG Scholar

Thiagarajar College of Engineering,

Department of Electrical and Electronics Engineering

E-mail: jeyadheepvignesh@gmail.com

Varatharajan M., Assistant Professor

Thiagarajar College of Engineering,

Department of Electrical and Electronics Engineering

E-mail: varatharajan@gmail.com

Rubina Sherrin M., PG Scholar

Thiagarajar College of Engineering,

Department of Electrical and Electronics Engineering

E-mail: rubinasherrin@gmail.com 